\title{
Detecting Maximum Inscribed Rectangle Based On Election Campaign Algorithm
}

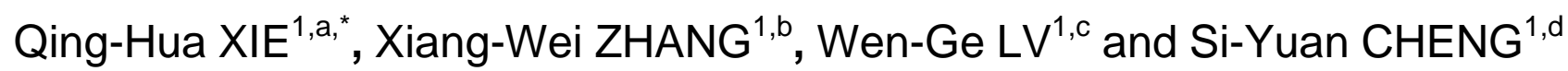 \\ ${ }^{1}$ School of Electromechanical Engineering, Guangdong University of Technology, Guangzhou china \\ axqhcool@163.com, ’xwzhang@gdut.edu.cn, 'Ivwenge@163.com, dimdesign@gdut.edu.cn \\ ${ }^{*}$ Corresponding author
}

Keywords: Maximum Inscribed Rectangle, Constrained Grid Graph, Election Campaign Algorithm, Optimization.

Abstract. Detecting maximum inscribed rectangle on irregularly shaped raw material is a key problem of cutting and nesting process in natural stone production plant. The present ruling method is the artificial realization, and has the disadvantages of low efficiency, poor utilization rate and so on. Based on the constrained gird graph and Election Campaign Algorithm, optimization mathematical model is used to detect the maximum inscribed rectangle. A new detection method is proposed and get the better utilization rate method for production. The experimental results show that the optimization method has a good applicability to the irregularly shape, and has a high operational characteristics to achieve the object of maximum inscribed rectangle detection with high precision.

\section{Introduction}

Stone slab is directly cut from the natural rock blocks. It has the irregular profile and various surface defects, such as the gall stone, holes, cracks and other defects ${ }^{[1]}$. It is difficult to get the best utilization rate of cutting and nesting stone slab, artificial realization is usually the solution of this problem ${ }^{[2,3]}$. Digital image processing technology provides a technical support to determine such as the minimum external rectangle and maximum inscribed rectangle parallel to the coordinate axes ${ }^{[4]}$. Detecting maximum inscribed rectangle on irregularly shaped is not a tradition image processing method. Also, the optimal layout and cutting of rectangular stone is feasible method to increase utilization rate of a rectangular material ${ }^{[5]}$. Get the maximum inscribed rectangle on irregularly shaped and then process the optimal layout and cutting will be the better utilization rate solution for natural stone production plant.

In this paper, we use constrained gird graph to detect the overlapping area of the object shape and test rectangle. The author uses the election campaign algorithm to find out the center and direction of the test rectangle which has the maximum area and no overlapping area with the object shape. After calibration, the digital images of objects with irregular shapes are collected by the camera, and the image is processed by the image correction mothed. The corrected images will be used to get the object shape and constrained gird graph. The next step, the optimization processing of mathematical model compute the closest solution, the defect of maximum inscribed rectangle complete.

\section{Constrained Gird Graph}

According to the actual use of stone cutting precision, we use virtual grid graphics witch has the spacing of required precision in the image. Each pixel of image is divide to equal virtual grid just like the sub pixel method ${ }^{[6]}$. A sample profile retrieval each virtual grid in the region, the definition of each gird is 0 , gird out of the region is not 0 . For example, the actual graphic discretization is shown in Figure 1. For the interpretation of clarity, we abstract some exceptions for sample enlarged graphics. If you can retrieve the picture element in the virtual grid area and small area, is defined as 0 , said the need to discharge at the corresponding position; the remaining non 0, as shown in Figure 1 (b). Fig. 1 (c) said there is a hole in the middle of the picture. All discharge can be considered as the target number is non-zero. 


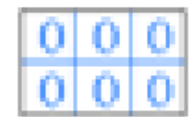

(a) Stencil 1

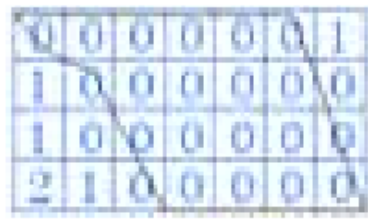

(b) Stencil 2

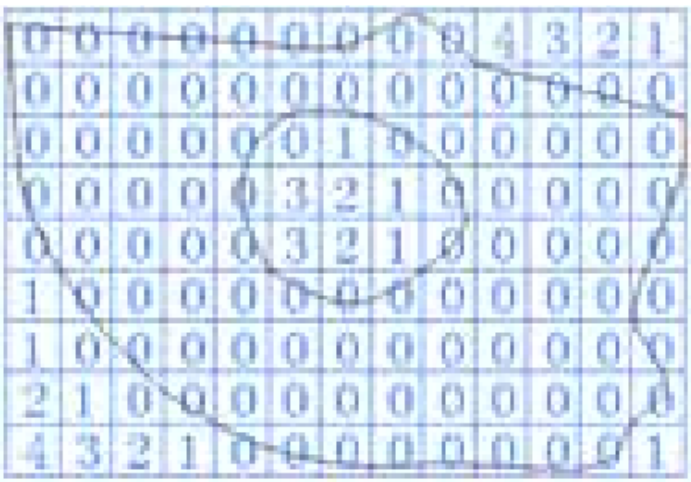

(c) Stencil 3

Fig. 1 Discrete grids and corresponding pixel values

As is shown in Figure 2, the images of stone slabs have has the irregular profile and various surface features. Figure 3 represent the constrained gird graphs. After discrete transformation processing, the images of stone slabs converted into minimum virtual rectangular grid graphs includes a set of grid information value.
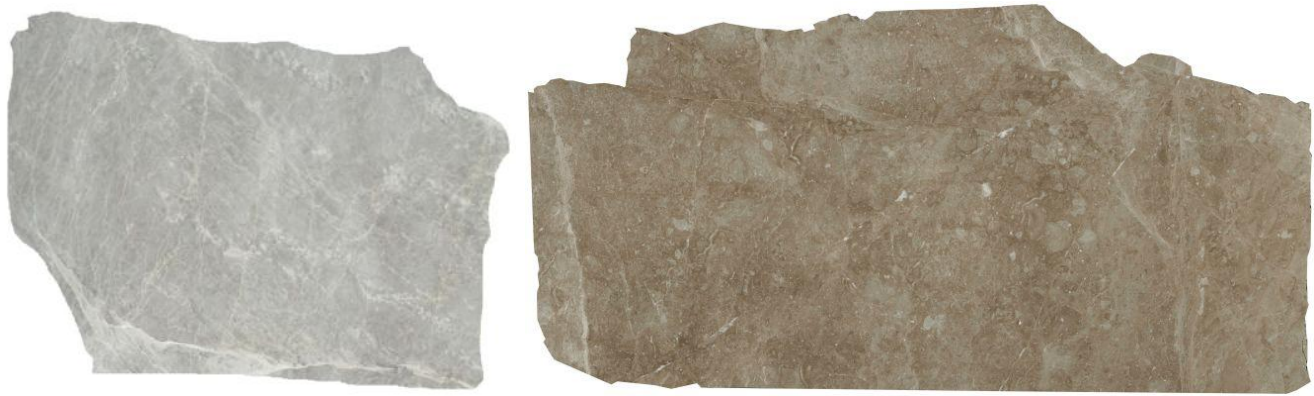

Fig. 2 Sample of Stone Slab

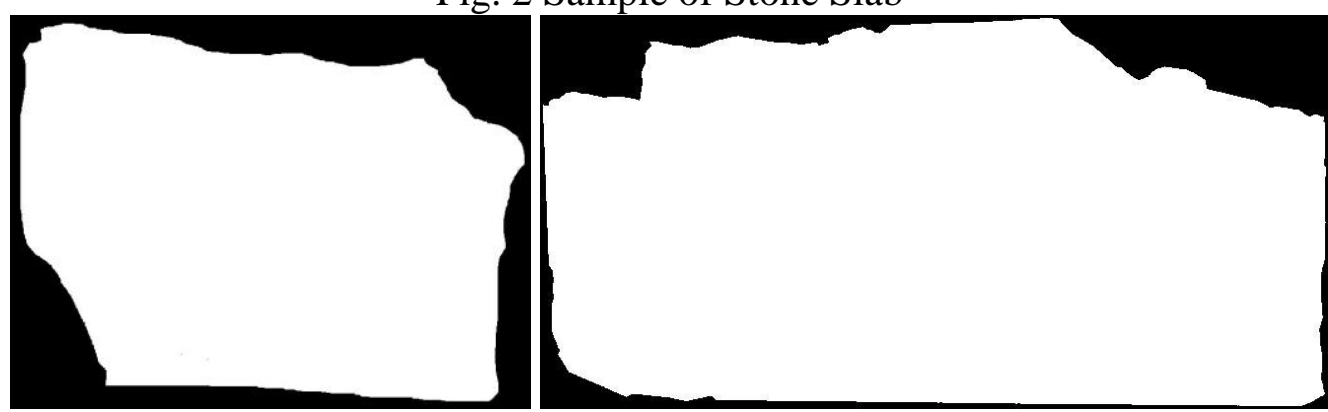

Fig. 3 Constrained Gird Graph of Stone Slab

\section{Optimization Processing Mathematical Model}

If the inscribed rectangle has the maximum area and has not overlapping area with the outside area of the object shape, the solution should be the area we detect for. The Mathematical Model describes as below.

$$
\left\{\begin{array}{c}
A=\max \left(w^{*} l\right) \\
S(r, c) \notin R \\
B(r, c, \theta) \in R
\end{array}\right.
$$

$B(r, c, \theta)$ is the gird at the border of the non-zero, $S(r, c)$ is the gird in the inscribed rectangle area, $A$ is the area measure of inscribed rectangle, the optimization program find the position on the border of the shape and detect an angle of the inscribed rectangle, then find the width and height which get the maximum area of the rectangle and test whether the overlapping area exists. 


\section{Get Border Gird}

2. Get Angle.

\section{Get Width and Height.}

4. Test Overlapping. The gird in the overlapping area has non-zero feature and change number in the border area.

5. Calculate the area measure. Select Width and Height, test overlapping then change to bigger number and test again.

\section{Election Campaign Algorithm}

Election campaign algorithm (ECA) is a new optimization algorithm simulating election process. Election candidates always pursue the maximum support from voters by means of various election behaviors. They get the overview of their support from the voters according to the sample survey, and then decide what to do on the next move. Solution space is imagined as voters and current solutions are imagined as candidates in ECA. The function value of a feasible solution is named as the prestige of a voter and the function value of a current solution is named as the prestige of a candidate.

In ECA, the candidates influence the voters round them, the voter's support is in proportion to the distance between the candidates and the voters. The voter will vote to the candidate which he pefer the most. The sum of location coordinates of every voters supported the candidate powered by its contribution is a new location coordinates, which is named support focus, it is the next position of the candidate. Such computational cycle is done continually until a candidate finds the position of the highest support, which is the global solution of the optimization problems.

Framework of the election campaign algorithm is described as the follows:

Set the Algorithm Parameters. Main parameters of Election campaign algorithm are:

- The number of candidates.

- The number of voters.

- The number of floating voters.

- Target accuracy.

Generate the Candidates and Calculate the Prestige of Them. Generated the define number of candidates in feasible solution field on the uniform distribution. Use the objective function to calculate the prestige of each candidate.

Generate the Voters. The uniform distribution is employed to generate the voters in feasible solution field.

Compute the Investigate Mean Square of Candidates. Higher prestige of a candidate, smaller the mean square deviation of local voters, so that ECA is able to converge to local optimization solution rapidly and steadily. The following formula is used to describe the relation of the prestige and the mean square deviation of a candidate.

$$
\sigma_{C_{i}}=\frac{\left(P_{\text {Max }}-P_{\mathrm{C}_{i}}\right)}{\left(P_{\text {Max }}-P_{\text {Min }}\right)}
$$

Where $\sigma_{C_{i}}$ represents the mean square deviation of candidate $C_{i} ; P_{\text {Max }}$ and $P_{\text {Min }}$ are the maximum and minimum prestige of candidates.

Next step, voters are generated around each candidate on the normal distribution.

$$
N_{C_{i}}=\frac{\left(P_{\text {Max }}-P_{\mathrm{C}_{i}}\right)}{\left(P_{\text {Max }}-P_{\text {Min }}\right)}\left(N_{V}-N_{F V}\right)
$$

$N_{C_{i}}$ is the voter number of candidate $C_{i}, N_{V}$ is the number of all voters, $N_{F V}$ is the number of floating voters. Floating voters are generated on the uniform distribution. Here, it is supposed that candidate's effect on voters declined linearly. 
Calculate the Support of the Voters. Candidates can influence the voters within their effect range, the effect on voter $V_{j}$ from candidate $C_{i}$ is

$$
F_{C_{i} V_{j}}=\frac{D_{C_{i} M a x}-D_{C_{i} V_{j}}}{D_{C_{i} \operatorname{Max}}} P_{C_{i}}
$$

$F_{C_{i} V_{j}}$ is the effect on voter $V_{j}$ form candidate $C_{i}, V_{j}$ is the global or local voter. Here, it is supposed that candidate's effect on voters declined linearly.

Then Compute the prestige of voters by means of objective function.

The support of a voter is proportional to his prestige, and then the proportional constant will be reduced, so the prestige of a voter can used to denote the support of a voter directly. A voter may be influenced by several candidates; the voter should distribute his support to candidates proportionally on the magnitude of effect from candidate to voter. The support from voter $V_{j}$ to candidate $C_{i}$ is

$$
S_{C_{i} V_{j}}=\frac{F_{C_{i} V_{j}}}{\sum_{i=1}^{m} \max \left(F_{C_{1} V_{j}}, F_{C_{2} V_{j}}, \cdots, F_{C_{i} V_{j}}, \cdots F_{C_{m} V_{j}}\right)} P_{V_{j}}
$$

$S_{C_{i} V_{j}}$ represents the support from the voter $V_{j}$ to candidate $C_{i}$.

Find the Support Focus of Candidates. A new position coordinate will achieve by means of summing the products of the support from the voters to the candidate and the position coordinate of the voters. It is named the support focus.

$$
x_{C_{i}}^{*}=\frac{\sum_{j=1}^{n} S_{C_{i} V_{j}} x_{V_{j}}}{\sum_{j=1}^{n} \max \left(S_{C_{i} V_{1}}, S_{C_{i} V_{2}}, \cdots, S_{C_{i} V_{j}}, \cdots S_{C_{i} V_{n}}\right)}
$$

$x_{C_{i}}^{*}$ is the support focus of the candidate $C_{i}$. The support focus of a candidate is obtained by investigating, which depends on those voters whose distances to the candidate are nearer and the prestige is higher relatively. The next post of the candidate should be the support focus, where the candidate will have the higher support.

Calculate the Prestige of the Candidates and Compare the Prestige of the Voters with the Candidates. In order to jump out of local optimization solution and increase search rate, the prestige of candidates are compared to that of voters, if the prestige of a voter is higher than that of a candidate, the voter with higher prestige will substitute for the candidate and the that candidate of lower prestige will be eliminated in election.

Check Whether the Condition is Reach. Check whether the condition is reach, otherwise return to step B to execute the period. Here, the condition could be the number of select candidates in the target accuracy or max cycle. Do that circularly until the highest support is found.

$$
\left|P_{C_{i}}^{t}-P_{C_{i}}^{t-1}\right| \leq \xi
$$

$P_{C_{i}}^{t}$ represents the prestige of candidate $C_{i}$ in cycle t. When the absolute value of the difference between the prestige of the candidates in the present cycle and the previous cycle less than or equal to the target accuracy $\xi$, the computation stop.

Do that circularly until the highest support is found.

Flow scheme of the election campaign algorithm is shown in Fig. 4. 


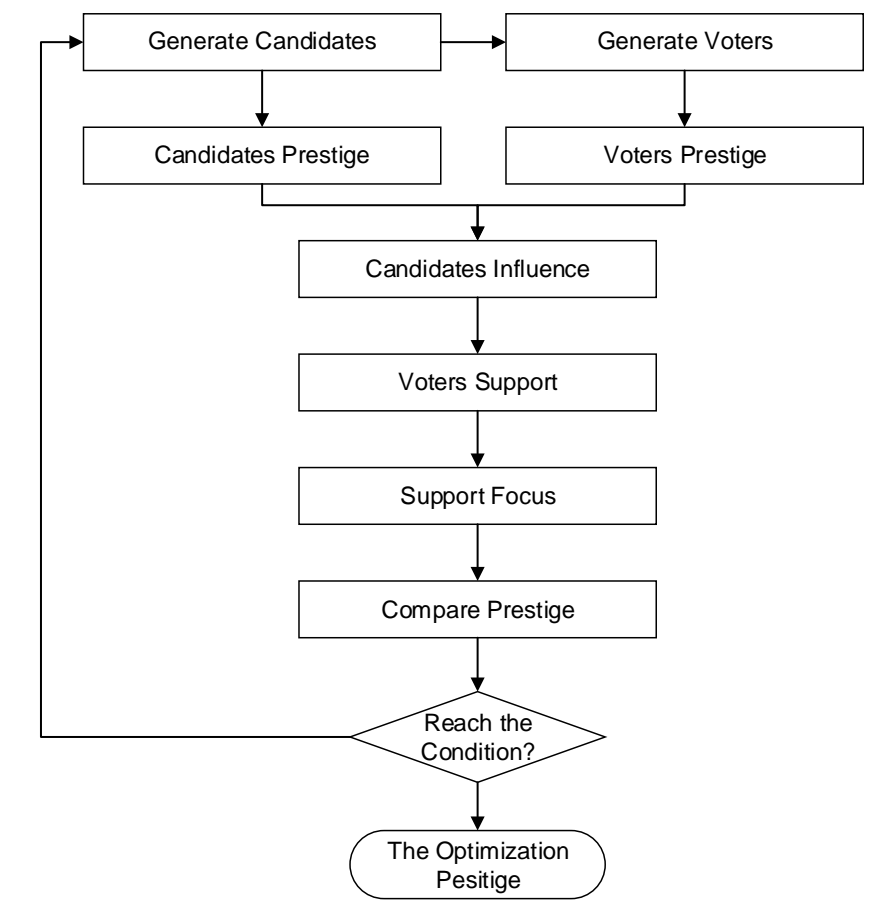

Fig. 4 Flow scheme of election campaign algorithm

\section{Sample of Detecting Maximum Inscribed Rectangle}

Start on the gird of shape border, then get angle, width and height, with no overlapping. In all these solution, find the one has the maximum area ${ }^{[7,8]}$.

Using Election campaign algorithm, the number of candidates define as 5, the number of voters is 10 , the number of floating voters is 5 , and the target accuracy is set as $1 \%$. The experiment get the maximum inscribed rectangle of the stone slab, as is show in Fig. 3, it prove the feasibility of the algorithm.

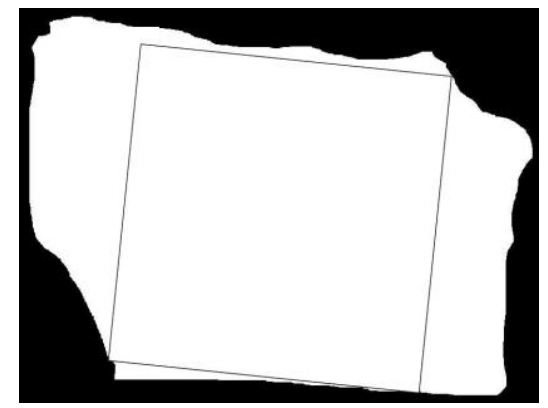

Fig. 5 Maximum Inscribed Rectangle of Stone Slab

\section{Conclusions}

With the method of the transformation from stone slab image to constrained gird graph, the overlapping of object shape and test rectangle can be detected, all the rectangle meet the conditions can be found and the one has maximum area will be detect by the processing of Election campaign algorithm optimization program, and reach the optimal solution. The disparity map obtained from the results can show that method is feasible.

Election campaign algorithm has good performance to avoid the solution trapped in local optima. This is particularly important when dealing with highly multimodal functions. For future work, another feature and different energy function will be used in the detecting of maximum inscribed rectangle. 


\section{References}

[1] Bennell JA, Dowsland KA, Dowsland WB. The irregular cutting-stock problem-A new procedure for deriving the no-fit polygon. Computers and Operations Research, 2001,28(3):271-287.

[2] Gomes AM, Oliveira JF. A 2-exchange heuristic for nesting problems. European Journal of Operational Research, 2002,141(3):359-370.

[3] Heistermann J, Lengauer T. The nesting problem in the leather manufacturing industry. Annals of Operational Research, 1995,57: 147-173.

[4] Chen TT, Chi DC, Liang Q. Multi-leaf area measurement method based on geometric correction with rectangular box. Journal of Agricultural Engineering, 2012; ( 4) : 206-213

[5] Lu R, Wang J B, Chen N N, et al. Fast algorithm for extracting minimum enclosing rectangle of target image. Computer Engineering, 2010; ( 3) : 178-180X.

[6] Ou W N. Analysis and comparison of several common edge detection operators. Modern Computer ( Pro) , 2010; ( 5) : 75-77

[7] Xu X J, Shao Y, Guo S F. Edge detection operators and their application in flame image. Micro Computer Information, 2008; ( 6) : 277-279

[8] Heckmann R, Lengauer T. Computing closely mathing upper and lower bounds on textile nesting problems. European Journal of Operational Research, 1999,108(3):473-489. 